\title{
Management of the palatally displaced canine
}

\author{
Philip Edward Benson ${ }^{1}$ Elizabeth Anne Graham² $•$ Nicola Ann Parkin ${ }^{3}$
}

Received: 13 June 2018 / Accepted: 18 September 2018 / Published online: 20 November 2018

(c) The Author(s) 2018

\begin{abstract}
The maxillary permanent canine is a common tooth to deviate from its normal path of eruption and become displaced, usually into the roof of the mouth or palate. This article outlines the assessment, diagnosis and potential early management options for a palatally displaced permanent canine tooth (PDC). Some adverse consequences of a PDC are discussed.
\end{abstract}

Keywords Orthodontics $\cdot$ Malocclusion $\cdot$ Canine $\cdot$ Displacement $\cdot$ Intervention

\section{Quick reference/description}

The maxillary permanent canine is a common tooth to deviate from its normal path of eruption and become displaced, usually into the roof of the mouth or palate. This occurs in about $2 \%$ of the population. There are various theories about why this might happen, but one predisposing factor is that the maxillary canine has a long path of eruption.

Early detection of a palatally displaced maxillary permanent canine (PDC), in a young person, is important if interceptive is to be undertaken at the ideal age (10-14 years). The aim of early treatment is to correct the path of eruption and prevent the need for more complicated treatment, namely surgical exposure and prolonged orthodontic alignment.

Philip Edward Benson

p.benson@sheffield.ac.uk

1 Academic Unit of Oral Health, Dentistry and Society, School of Clinical Dentistry, University of Sheffield, Sheffield S10 2TA, UK

2 Orthodontic Department, Dorset County Hospital NHS Foundation Trust, Dorchester DT1 2JY, UK

3 Orthodontic Department, Charles Clifford Dental Hospital, Sheffield Teaching Hospitals NHS Foundation Trust, Sheffield S10 2SZ, UK 


\section{Assessment and diagnosis}

The diagnosis of a PDC is initially undertaken through a clinical examination. Although there can be considerable variation in the chronological age of a young person compared with their dental age, generally, if the permanent canine is not palpable in the buccal sulcus by the age 11 years, then a radiograph should be taken. The distance of the unerupted canine from the occlusal plane, the angulation and in particular the medial position should be assessed.

\section{Management}

The management of an unerupted canine will depend on the findings of the clinical and radiographic examination, as well as the expectations, cooperation and age of the patient. The best time to intervene is between the ages of 10 and 14 years (Table 1). Beyond the age the permanent canine would normally be expected to erupt; the outcome of any intervention is likely to be even less predictable.

\section{Overview}

See Table 1.

\section{Procedure}

\section{Extraction of the primary canine}

Several studies have indicated that extraction of the primary canine in young people might lead to improvement in the position of the permanent canine; however, this can be inconsistent and we need more information about when this intervention is likely to help.

\section{Space creation}

When the permanent canine is displaced and still has some eruptive potential, then space can be created using transverse expansion of the maxillary arch with rapid maxillary expansion (RME) or anterior-posterior expansion, using a bonded orthodontic appliance or headgear.

\section{Rapid maxillary expansion (RME) (Fig. 1)}

Expansion is continued until the palatal cusps of the maxillary posterior teeth are in contact with the buccal cusps of the mandibular posterior teeth.

RME can be followed by placement of a transpalatal arch (TPA) and extraction of the primary canine if needed. 


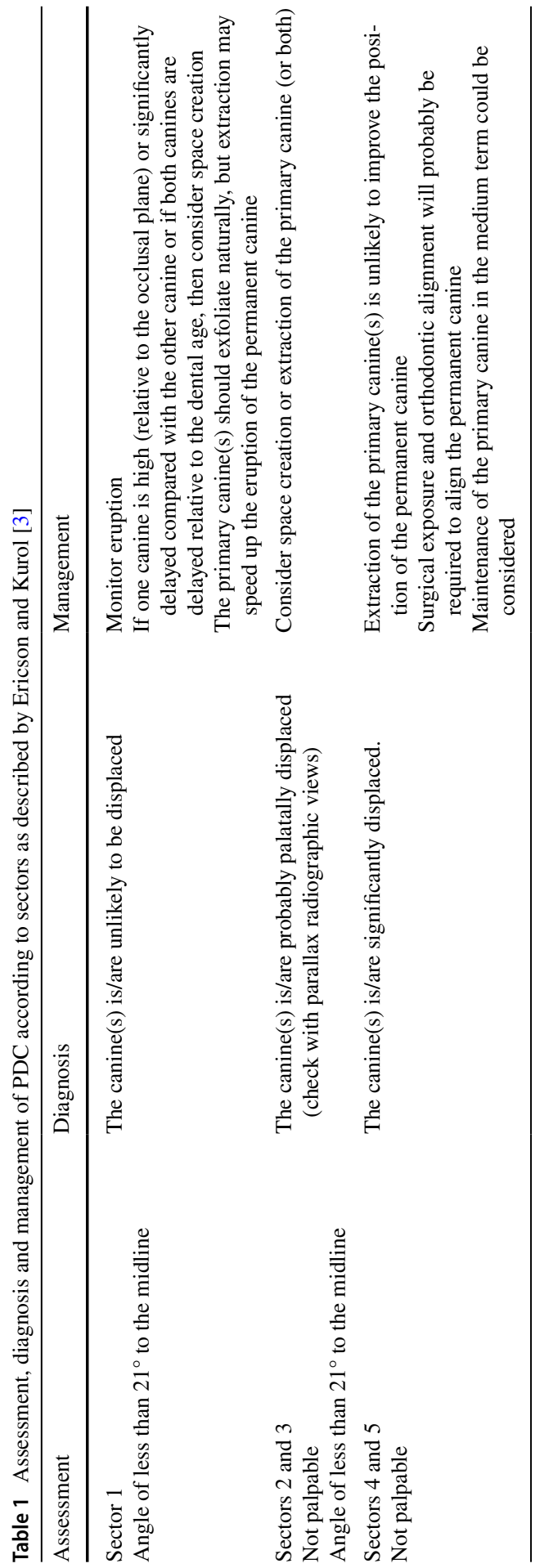



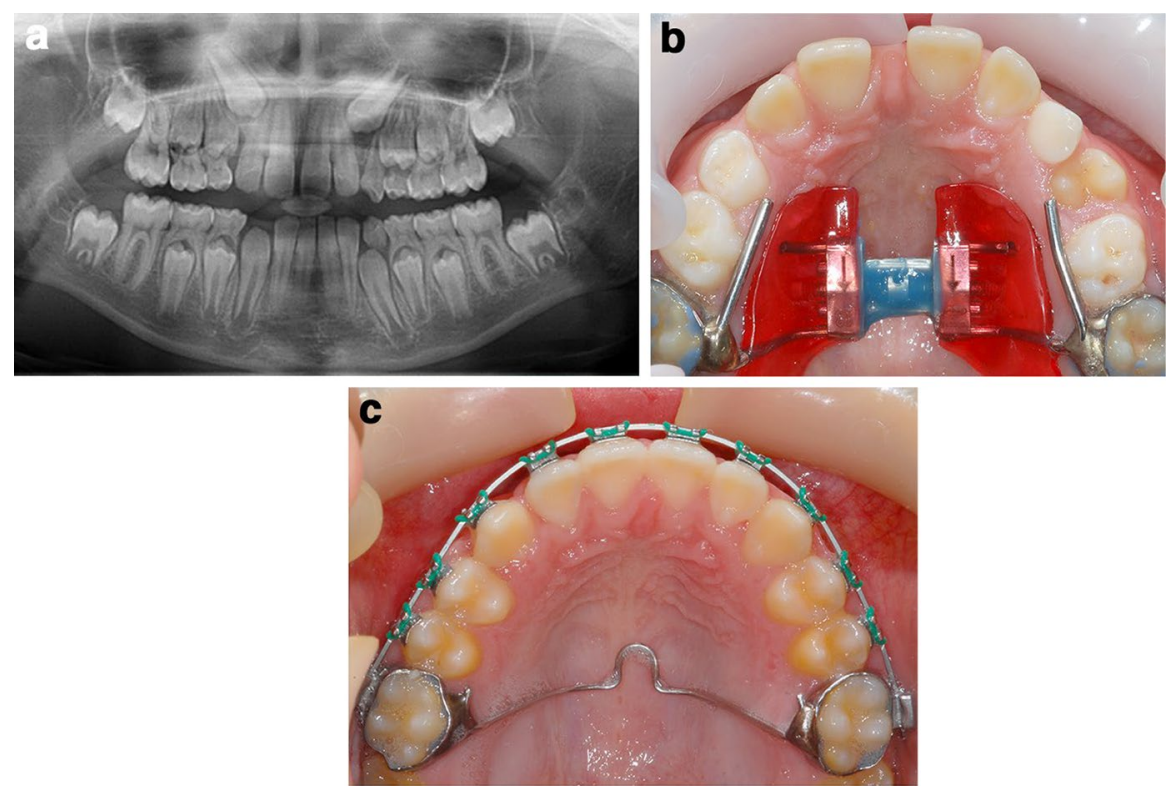

Fig. 1 a Pretreatment records with insufficient space for the maxillary canines and a displaced upper right permanent canine. b Treatment with RME initially to widen the maxillary arch and create space. Eruption of the canine on the left 7 months after completion of expansion. c Appearance 25 months after expansion. Eruption of canine on the right after 20 months of treatment with fixed appliances. Surgical exposure is not required
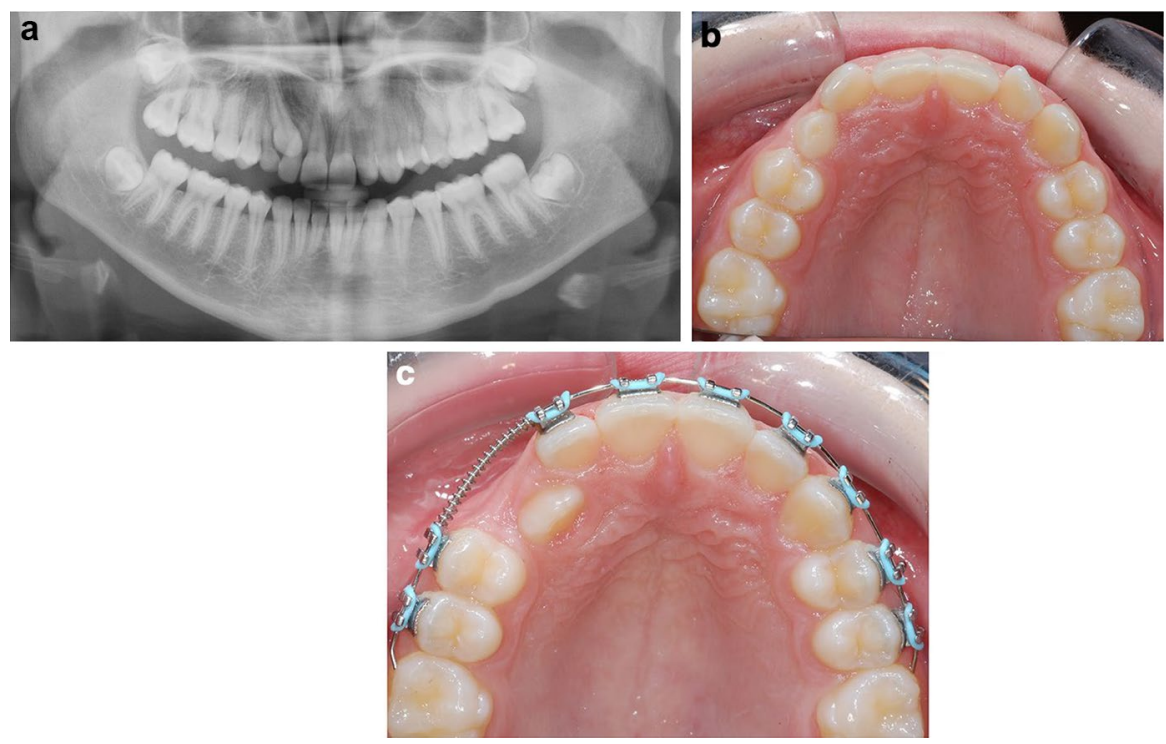

Fig. 2 An example of an impacted upper right canine. $1 \mathrm{~cm}$ of space is created using an upper fixed appliance. After that the permanent canine starts erupting. Surgery is not required 


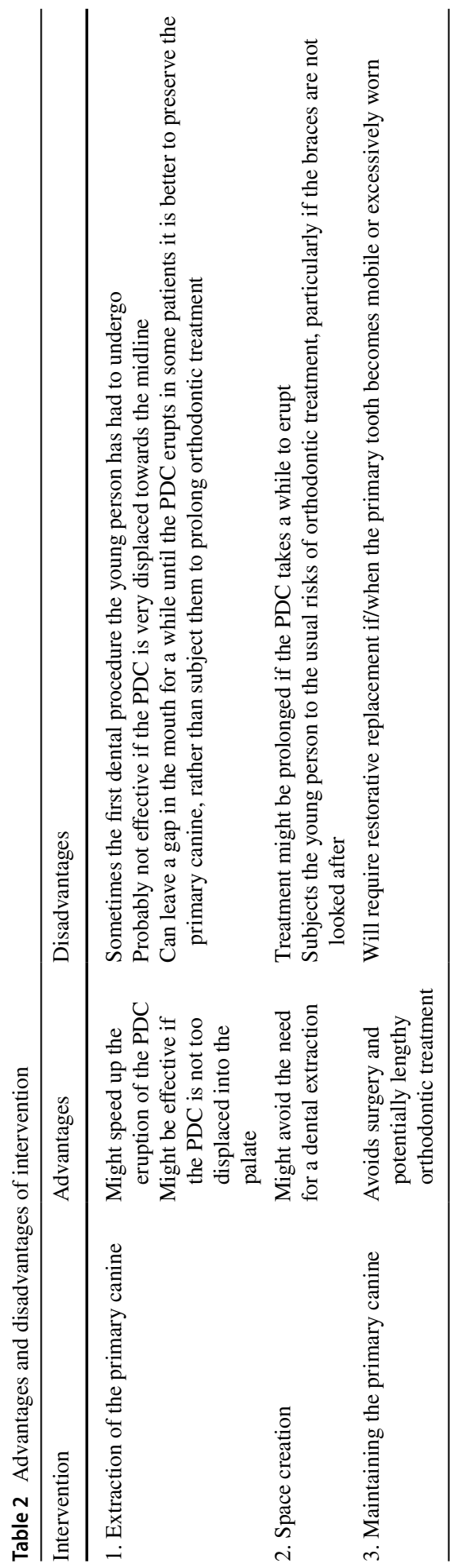


a
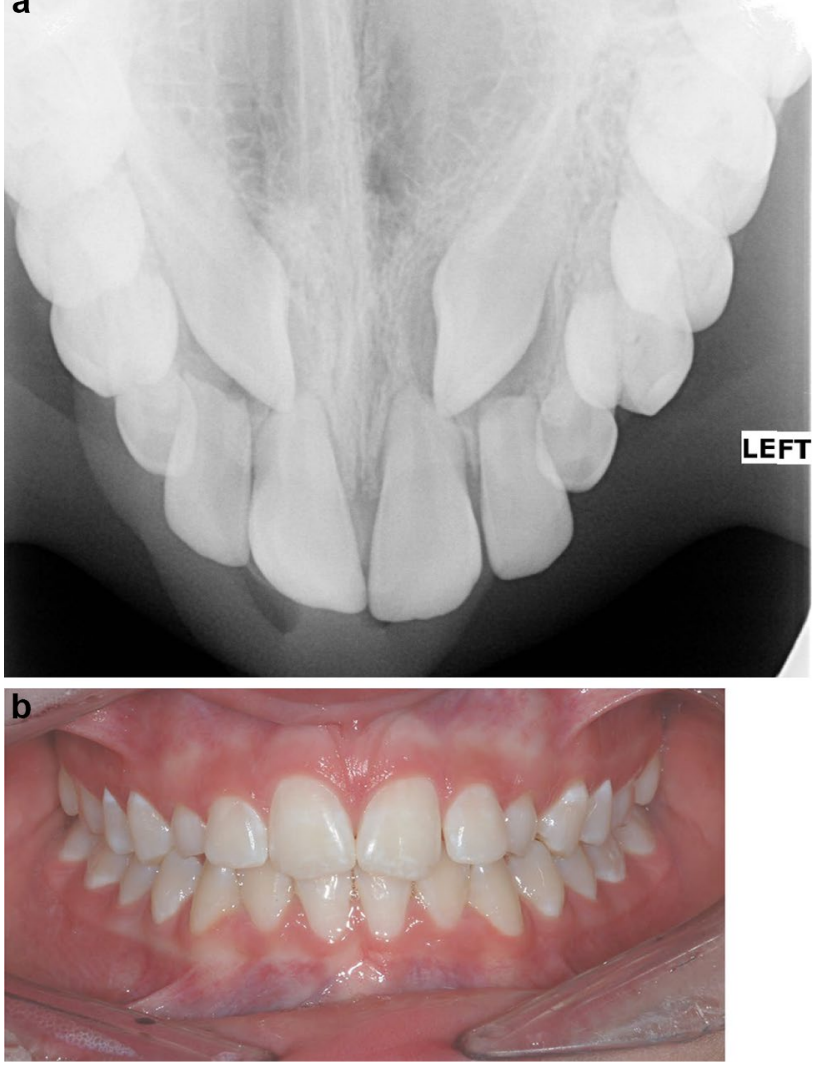

Fig. 3 a Standard maxillary occlusal radiograph with resorbed lateral and central incisors. b There are no clinical signs of resorption, no discoloration or mobility of any of the incisors

\section{Bonded orthodontic appliances (Fig. 2)}

Space can be created with bonded appliances, accepting an increased overjet and a shift of the upper centerline.

\section{Headgear}

Cervical pull headgear could be used in patients with moderate crowding and a class II tendency.

\section{Maintenance of the primary tooth}

If the permanent canine is considerably displaced from the line of the arch, maintenance of the primary tooth in the medium term, with or without restorative build-up, can be considered. 

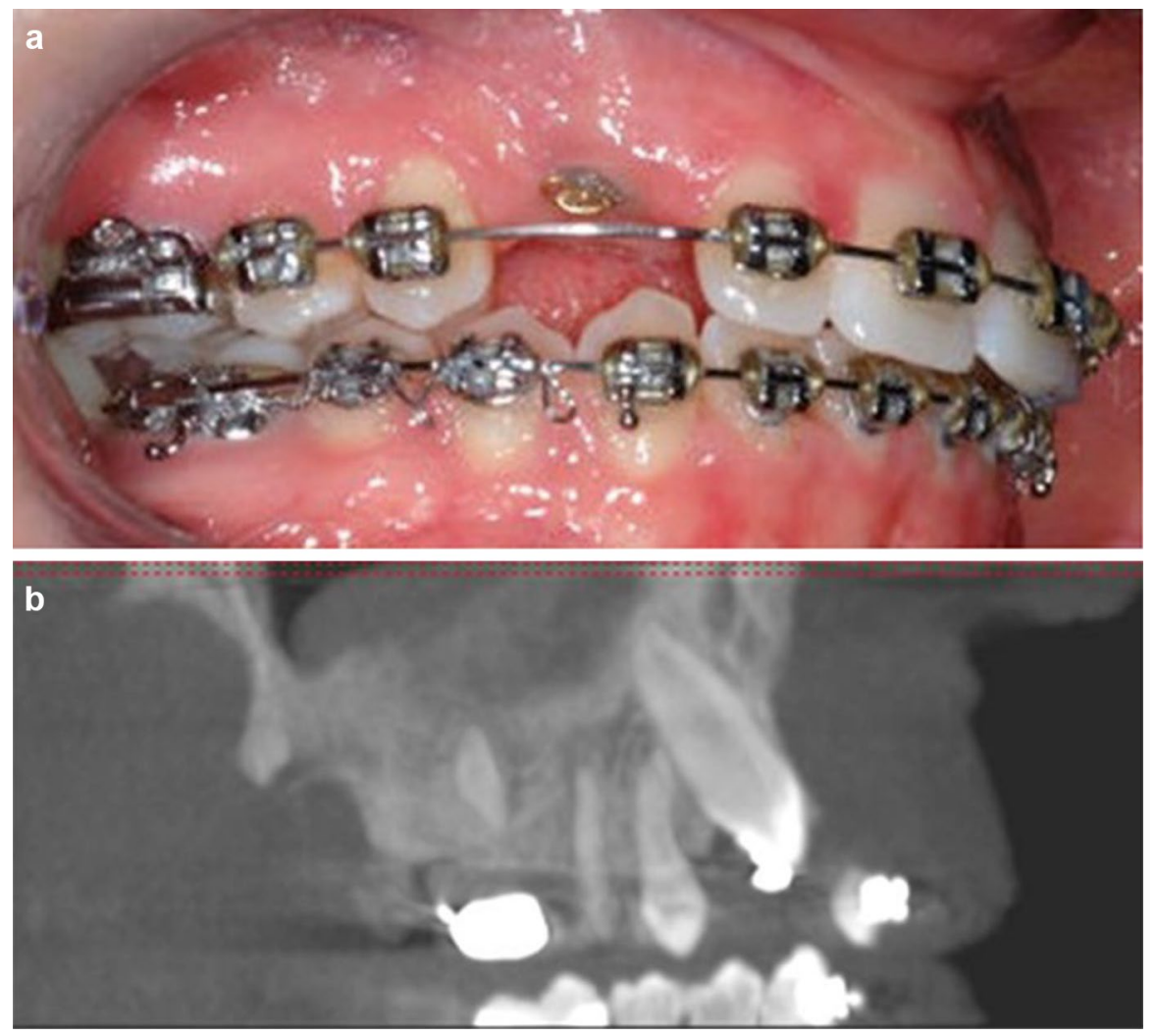

Fig. 4 a An unerupted maxillary canine that stopped moving in response to orthodontic forces. b The report of the $3 \mathrm{D}$ radiographic imaging stated that 'The upper right canine is impacted against the palatal surface of the root of the adjacent lateral incisor. It is possible to identify a periodontal ligament space around the buccal surface of the root, indicating no ankyloses, however there is an element of uncertainty with visualization of PDL owing to resolution limitations'

\section{Advantages and disadvantages of intervention (Table 2)}

\section{Pitfalls and complications}

A PDC can lead to resorption of adjacent teeth (Fig. 3).

Occasionally, the unerupted canine will not respond to orthodontic forces, either due to a true ankylosis (which is probably rare in the younger patient and with a careful surgical exposure) or to pseudoankylosis, when the unerupted tooth impacts against the root of an adjacent tooth or the buccal plate (Fig. 4).

Open Access This article is distributed under the terms of the Creative Commons Attribution 4.0 International License (http://creativecommons.org/licenses/by/4.0/), which permits unrestricted use, distribution, 
and reproduction in any medium, provided you give appropriate credit to the original author(s) and the source, provide a link to the Creative Commons license, and indicate if changes were made.

\section{Further reading}

1. Cobourne MT (ed) (2017) Orthodontic management of the developing dentition, early management of the palatally displaced maxillary permanent canine. Springer, Berlin. https://doi.org/10.1007/9783-319-54637-7_8

2. Ericson S, Kurol J (1986) Longitudinal study and analysis of clinical supervision of maxillary canine eruption. Commun Dent Oral Epidemiol 14(3):172-176

3. Ericson S, Kurol J (1988) Early treatment of palatally erupting maxillary canines by extraction of the primary canines. Eur J Orthod 10(4):283-295

4. Parkin N, Furness S, Shah A, Thind B, Marshman Z, Glenroy G, Dyer F, Benson PE (2012) Extraction of primary (baby) teeth for unerupted palatally displaced permanent canine teeth in children. Cochrane Database Syst Rev 12:CD004621. https://doi.org/10.1002/14651858.CD004621.pub3

5. Chaushu S, Kaczor-Urbanowicz K, Zadurska M, Becker A (2015) Predisposing factors for severe incisor root resorption associated with impacted maxillary canines. Am J Orthod Dentofac Orthop 147(1):52-60. https://doi.org/10.1016/j.ajodo.2014.09.012 